\title{
Identification of novel prophage regions in Xenorhabdus nematophila genome and gene expression analysis during phage-like particle induction
}

\author{
Emilie Lefoulon $^{1}$, Natalie Campbell ${ }^{1}$, S. Patricia Stock ${ }^{\text {Corresp. 1,2 }}$ \\ ${ }^{1}$ School of Animal and Comparative Biomedical Sciences, University of Arizona, Tucson, AZ 85721, USA \\ 2 College of Agriculture, California State University, Chico, CA 95929, USA \\ Corresponding Author: S. Patricia Stock \\ Email address: spstock@csuchico.edu
}

Background Entomopathogenic Xenorhabdus bacteria are endosymbionts of Steinernema nematodes and together they form an insecticidal mutualistic association that infects a wide range of insect species. Xenorhabdus produce an arsenal of toxins and secondary metabolites that kill the insect host. In addition, they can induce the production of diverse phage particles. A few studies have focused on one integrated phage responsible for producing a phage tail-like bacteriocin, associated with an antimicrobial activity against other Xenorhabdus species. However, very little is known about the diversity of prophage regions in Xenorhabdus species.

Methods In the present study, we identified several prophage regions in the genome of Xenorhabdus nematophila AN6/1. We performed a preliminary study on the relative expression of genes in these prophage regions. We also investigated some genes (not contained in prophage region) known to be involved in SOS bacterial response (recA and lexA) associated with mitomycin C and UV exposure.

Results We described two integrated prophage regions (designated Xnp3 and Xnp4) not previously described in the genome of Xenorhabdus nematophila AN6/1. The Xnp3 prophage region appears very similar to complete Mu-like bacteriophage. These prophages regions are not unique to $X$. nematophila species, although they appear less conserved among Xenorhabdus species when compared to the previously described p1 prophage region. Our results showed that mitomycin exposure induced an upregulation of recA and lexA suggesting activation of SOS response. In addition, mitomycin and UV exposure seems to lead to up-regulation of genes in three of the four integrated prophages regions. 
1 Identification of novel prophage regions in Xenorhabdus nematophila genome and gene 2 expression analysis during phage-like particle induction

3

4 Emilie Lefoulon ${ }^{1}$, Natalie Campbell ${ }^{1}$ and S. Patricia Stock ${ }^{1,2 *}$

5

$6{ }^{1}$ School of Animal and Comparative Biomedical Sciences, University of Arizona, Tucson, 7 Arizona, USA

$8{ }^{2}$ College of Agriculture, California State University Chico, Chico, California, USA

$11 *$ Corresponding author:

12 S. Patricia Stock

13 College of Agriculture, California State University Chico. $400 \mathrm{~W} 1^{\text {st }}$ Street, Chico, CA 95929, 14 USA

15 e-mail: spstock@csuchico.edu 
18 Abstract (500-word max)

19 Background

20 Entomopathogenic Xenorhabdus bacteria are endosymbionts of Steinernema nematodes and 21 together they form an insecticidal mutualistic association that infects a wide range of insect 22 species. Xenorhabdus produce an arsenal of toxins and secondary metabolites that kill the insect 23 host. In addition, they can induce the production of diverse phage particles. A few studies have 24 focused on one integrated phage responsible for producing a phage tail-like bacteriocin, associated 25 with an antimicrobial activity against other Xenorhabdus species. However, very little is known 26 about the diversity of prophage regions in Xenorhabdus species.

\section{Methods}

28 In the present study, we identified several prophage regions in the genome of Xenorhabdus 29 nematophila AN6/1. We performed a preliminary study on the relative expression of genes in these 30 prophage regions. We also investigated some genes (not contained in prophage region) known to 31 be involved in SOS bacterial response ( $\operatorname{rec} A$ and $\operatorname{lex} A$ ) associated with mitomycin $\mathrm{C}$ and $\mathrm{UV}$ 32 exposure.

\section{Results}

34 We described two integrated prophage regions (designated Xnp3 and Xnp4) not previously 35 described in the genome of Xenorhabdus nematophila AN6/1. The Xnp3 prophage region appears 36 very similar to complete Mu-like bacteriophage. These prophages regions are not unique to $X$. nematophila species, although they appear less conserved among Xenorhabdus species when compared to the previously described $\mathrm{p} 1$ prophage region. Our results showed that mitomycin $\mathrm{C}$ exposure induced an up-regulation of $r e c A$ and $\operatorname{lex} A$ suggesting activation of SOS response. In addition, mitomycin $\mathrm{C}$ and UV exposure seems to lead to up-regulation of genes in three of the four integrated prophages regions.

\section{Introduction}

44 Bacteria employ diverse strategies to occupy different ecological niches and outcompete 45 distantly related or kin bacteria (Griffin et al., 2004). Among them, is bacteriophage DNA, 46 (prophages or their remnants) which is integrated in bacterial genomes (Vacheron et al., 2021). 47 These prophage regions present costs and benefits. Potential costs can be the re-entry into the lytic 
48 cycle of the phage causing the bacterial death, or energy costs associate with the maintenance of 49 additional genetic material prophages for the bacteria (Harrison and Brockhurst, 2017; Selva et al., 50 2009). However, prophages can be beneficial by adding diversity to the bacterial gene repertoires 51 or act as weapons for bacterial competition (Harrison and Brockhurst, 2017). Indeed, prophages 52 can be co-opted by bacteria for production of derivatives or phages, such as phage-like 53 bacteriocins (Bobay et al., 2014; Vacheron et al., 2021).

54 Xenorhabdus spp. are intriguing bacteria with a dual lifecycle, they are endosymbionts of 55 entomopathogenic Steinernema nematodes and together they form a pathogenic partnership that 56 kills a wide range of insect species. The infective juvenile stages of the nematodes harbor bacteria 57 in an intestinal receptacle protecting them from the external environment (Flores-Lara et al., 2007; 58 Kim et al., 2012), and vector them from one insect host to another (Boemare, 2002). Once inside 59 an insect host, Steinernema nematodes release Xenorhabdus symbionts, which produce 60 antimicrobial secondary metabolites that preserve the cadaver from decomposing allowing the 61 nematodes to reproduce and complete their life cycle (Stock, 2019; Stock and Blair, 2008).

62 The arsenal of toxins produced by Xenorhabdus is not limited to secondary metabolites. The 63 production of phage-like particles were reported for the first time by Poinar (1980) and described 64 as "defective phages" composed of an inner core and a contractile sheath caring a base plate 65 (Poinar et al., 1980). Studies showed that these tail-like phage particles (named xenorhabdicin) 66 exhibit bactericidal activity against closely related bacterial species (Poinar et al., 1980; Thaler et 67 al., 1995). It has been suggested that these xenorhabdicins may constitute another antimicrobial 68 barrier to maintain the symbiotic association (Thaler et al., 1995) or might be involved in interspecies competition (Morales-Soto and Forst, 2011). Although, diverse phage particles were 70 also described in Xenorhabdus nematophila (Boemare et al., 1992; Thaler et al., 1995), most of 72 the research has focused on xenorhabdicins (Ciezki et al., 2017; Morales-Soto and Forst, 2011; Thappeta et al., 2020). Studies showed that a particular prophage region integrated in Xenorhabdus spp. genomes is involved in the induction of these xenorhabdicin (Morales-Soto et al., 2012; Morales-Soto and Forst, 2011). Two distinct prophages regions have been described in the genomes of $X$. nematophila and Xenorhabdus bovienii: one of these regions "p1 prophage" ( respectively $x n p 1$ or $x b p 1$ ) contains tail synthesis genes but lacks genes for capsid synthesis and replication, and the second region named p2 (xnp2 or xbp2) is similar to an intact $\mathrm{P} 2$ prophage 
79 to be up-regulated by exposure to mitomycin C (Morales-Soto and Forst, 2011). In addition, in $X$.

80 bovienii and $X$. nematophila inactivation of the sheath gene of the xnpl or xbpl region results in 81 the loss of phage tail-like bacteriocin activity (Morales-Soto and Forst, 2011). Although it has been 82 suggested that $X$. bovienii carries several other prophage clusters (more similar to lambda and $M u$ 83 prophage) (Ciezki et al., 2017) no further investigations have been performed to confirm this 84 statement. Herein, we focus on the discovery of other prophage regions in of $X$. nematophila 85 AN6/1 genome and conducted a preliminary study on the expression of in different prophage 86 regions during mitomycin $\mathrm{C}$ and $\mathrm{UV}$ exposure. We choose to compare the effect of the mitomycin 87 C the study of Xenorhabdus prophage regions (Boemare et al., 1992; Ciezki et al., 2017; Morales88 Soto and Forst, 2011; Thaler et al., 1995; Thappeta et al., 2020) and, the effect of UV which has 89 been previously used to induced phage particles on other bacteria (such as Escherichia coli) 90 (Baluch and Sussman, 1978; Takebe et al., 1967).

\section{Materials and Methods}

\section{Prophage region analysis}

94 Prophage regions present in the genomes of Xenorhabdus spp. were identified using PHASTER 95 (PHAge Search Tool Enhanced Release) (Arndt et al., 2016). PHASTER classified and detected 96 prophage region by completeness (incomplete, questionable and intact) thus we focused on 97 regions with higher score detected as "intact" (Arndt et al., 2016). Annotation of the proteins 98 localized in these regions were confirm by homology searches between the protein sequences using blastp (Camacho et al., 2009) and hidden Markov Models (HMM) profile using hmmscan in the HMMER web server (Potter et al., 2018).

\section{Bacterial strain, culture conditions and mitomycin $\mathrm{C}$-induced or $\mathrm{UV}$-induced cultures}

102

103 104

105

Cultures of Xenorhabdus nematophila strain AN6/1 were considered in the current study that are maintained in P. Stock's laboratory (University of Arizona). The bacteria were grown in $10 \mathrm{~mL}$ Luria Bertani (LB) broth supplemented with $0.1 \%(\mathrm{w} / \mathrm{v})$ sodium pyruvate (LBP) in a $50 \mathrm{ml}$ centrifuge tube with agitation at $28^{\circ} \mathrm{C}$ overnight for $12-16 \mathrm{~h}$. Culture growth was monitored based on $600 \mathrm{~nm}$ optical density (OD600). Then, the bacteria were subculture (1:5 dilution) and grown at $28^{\circ} \mathrm{C}$ to reach OD600 of $0.4-0.5$. Two types of induction were tested: a) with mitomycin $\mathrm{C}$ at a concentration of $5 \mu \mathrm{g} \mathrm{ml}^{-1}$ and b) $10 \mathrm{~min}$ exposure to UV $6 \mu \mathrm{J} / \mathrm{cm}^{2}$. Negative controls consisted of cultures not induced. Both induced and uninduced cultures were incubated at $28^{\circ} \mathrm{C}$ in an incubator 
110 with moderate shaking (1g). Two independent experiments were performed to assess: 1) late effect

111 of induction with two studied time points: $2 \mathrm{~h}$ and $10 \mathrm{~h}$ after induction, and 2) early effect of

112 mitomycin $\mathrm{C}$ induction with two studied time points: $15 \mathrm{~min}$ and $40 \mathrm{~min}$ after induction. For both

113 experiments, OD600 was quantified at each time point and results were plotted using scatterplot

114 in R environment (Fox and Weisberg, 2019).

115 RNA extraction and RT-PCR

116 At each time point, three replicates of $1.5 \mathrm{~mL}$ of cultures were collected, centrifugated at $3,080 \mathrm{~g}$

117 for $10 \mathrm{~min}$. The supernatant was discarded and $750 \mu \mathrm{L}$ Trizol was added to the pellet. Then, 150

$118 \mu \mathrm{L}$ chloroform was added to the bacteria mix and incubated on ice $15 \mathrm{~min}$. After centrifugation at $119\left(20,821 \mathrm{~g}\right.$ for $15 \mathrm{~min}$ at $\left.4^{\circ} \mathrm{C}\right)$ the aqueous phase was transferred to a new tube and $375 \mu \mathrm{L}$ of 120 isopropanol were added. After $10 \mathrm{~min}$ of incubation on ice, samples were centrifuge for $10 \mathrm{~min}$ at $12120,821 \mathrm{~g}$ at $4{ }^{\circ} \mathrm{C}$. The supernatant was discarded, and $1 \mathrm{~mL}$ of $75 \%$ ethanol was added to rinse the 122 samples which were then vortexed, followed by centrifugation for $5 \mathrm{~min}$ at $7,885 \mathrm{~g}$ at $4^{\circ} \mathrm{C}$. The 123 rinse step was repeated, and the RNA was air-dry and resuspend in water RNase-free. An 124 additional incubation step for $10 \mathrm{~min}$ at $55^{\circ} \mathrm{C}$ was considered to improve yield of the RNA elution.

125 The RNA was quantified using a Nanodrop and $300 \mathrm{ng}$ of RNA was used as the template for cDNA 126 synthesis. cDNA was synthesized using the Bioline SensiFast cDNA Synthesis kit following 127 manufacturer's protocols.

\section{8 qPCR of prophage region genes}

129 Quantitative real-time polymerase chain reaction (qRT-PCR) was used to measure the relative 130 gene expression across induction conditions. Each primer set condition was optimized using 131 Bioline SensiFast No ROX Sybr Master Mix and summarized in the S1 Table. Primers were 132 designed based on the genome reference of Xenorhabdus nematophila AN6/1 (NZ_LN681227) 133 using AmplifX (v2.0.7) (Nicolas Jullien; https://inp.univ-amu.fr/en/). A total of 10 pairs primers 134 were used in this study (S1 Table). We normalized the gene expression using the DNA gyrase 135 subunit A, gyrA as housekeeping gene. We used the Pfaffl model to calculate the relative gene 136 expression using the formula : $\frac{\left(E_{G O I)}{ }^{\Delta C t G O I}\right.}{\left(E_{R e f}\right)^{\Delta C t R e f}}($ Pfaffl, 2001). Primer efficiency was determined for each 137 gene by running a standard curve and converted primers efficiency $(\mathrm{E})$ was calculated as follows: 138 (primer efficiency $(\%) / 100)+1$. The calculated $\mathrm{Ct}$ from uninduced cultures was used as a 139 calibrator when calculating the $\Delta \mathrm{Ct}$ values for all the samples. The relative gene expression values 
140 were transformed by a logarithmic base 2 function to be plot as Boxplot in $\mathrm{R}$ environment. The

141 distribution of the data was not normal, thus the variance of the relative expression with the non-

142 parametric Kruskal-wallis rank sum associated with multiple pairwise comparisons was

143 considered using the Dunn's test in the R environment (dunn.test package) (Dunn, 1964).

144 Phage particles preparation and SDS-PAGE gels

145 Three time points were selected to purified induced phage particles: the two time points of the 146 early induction experiment, 15 and $40 \mathrm{~min}$ after induction, and the last time point of the late 147 induction experiment (10 h after induction). A total of $8 \mathrm{~mL}$ of induced and uninduced cultures 148 were centrifuged at 3,080g for $10 \mathrm{~min}$ to remove cellular debris. The supernatants were filtered 149 using $0.22 \mu \mathrm{m}$ pore size filter. Then, $\mathrm{NaCl}$ and polyethylene glycol 8000 (PEG 8000) solution were 150 added to reach respectively $1 \mathrm{M}$ and $10 \%(\mathrm{wt} / \mathrm{vol})$ final concentration to precipitate the phage 151 particles. The solution was incubated overnight at $4{ }^{\circ} \mathrm{C}$. The particles were collected by 152 centrifugation of $27,720 \mathrm{~g} 30 \mathrm{~min}$ at $4^{\circ} \mathrm{C}$ and suspended in $200 \mu \mathrm{L}$ in LBP. Manufacturer's 153 instructions were followed for the electrophoresis of phage particle samples using the Mini154 PROTEAN TGXTM precast gels system (Bio-Rad), followed by Coomassie blue staining to 155 visualize precipitated proteins.

156

\section{Results}

158 Four intact prophage regions are present in genomes of $X$. nematophila strain AN6/1

159 Of the 13 prophage regions identified by PHASTER in the studied genomes of $X$. nematophila 160 strain AN6/1 (NZ_LN681227), only four were detected as potential "intact" prophage regions.

161 Two of them are the prophage regions previously described: Xnp1 (localized at $1.05 \mathrm{M}$ to $1.09 \mathrm{M}$ ), 162 the P2-like remnant phage previously described as responsible of bacteriocin production; Xnp2 163 (localized at 3.03M to $3.06 \mathrm{M}$ ), the P2-like complete phage previously described as not involved 164 in bacteriocin production (Figure 1). These two regions display similarities with P2-like 165 bacteriophages. Our analysis also suggests strong similarities with the bacteriophage RE-2010 166 isolated from Salmonella enterica Serovar Enteritidis strain LK5 (NC_019488). As previously 167 described, the prophage region xnp1 is characterized by the absence of genes coding head capsids 168 proteins, as well as portal proteins or terminases. Another difference between the two P2-like 
169 prophages, is the region containing genes responsible to lysogeny control: Xnp2 region exhibited

$170 \mathrm{C}$ immunity repressor and Cox protein while Xnp1 exhibited CI repressor (Figure 1; S3 Table).

171 The two newly identified prophage regions are "intact" regions and are designated as Xnp3

172 and Xnp4 to aligned with previously used nomenclature. Region Xnp3 (localized at 3.28M to

$1733.35 \mathrm{M}$ ) presents strong similarities with Mu-like phage, while region Xnp4 (localized at 1.86M to

$1741.90 \mathrm{M}$ ) is a remnant phage presenting similarities with Siphoviridae-like bacteriophages (Figure

175 1). Compared to the other prophage regions, the Xnp4 region contained higher number of 176 transposases and insertion sequence elements (IS), few phage-related proteins were clearly 177 identified (only tail proteins) and no regulator or repressor protein was detected (Figure1; S3 178 Table).

179 Our analysis also suggests strong similarities between Xnp3 and the SfMu bacteriophage 180 isolated from Shigella flexneri (NC_027382) and the D108 bacteriophage isolated from 181 Escherichia coli (NC_013594). PHASTER also detected another prophage region of 67.4Kb with 182 two distinct prophage regions: 1) a $33.4 \mathrm{~kb}$ region (localized at $3.283 \mathrm{M}$ to $3.316 \mathrm{M}$ ) which is like a 183 complete $\mathrm{Mu}$-like bacteriophage and 2) a 22.6kb region (localized at 3.319M to 3.341M) which 184 seems to be a remnant prophage (Figure 1; S3 Table). We focused on the first region (like Mu-like 185 phage), which when compared to closely related bacteriophages, lacks the terminal part including 186 one tail collar-fiber protein (S), as well as the DNA-binding transcriptional regulator Com and 187 mom proteins (Figure 2). This prophage region is not only present in $X$. nematophila but seems to 188 be present (or has similar prophage regions) in the three other Xenorhabdus species including: 189 Xenorhabdus cabanillasii strain JM26, Xenorhabdus miraniensis strain DSM17902 and 190 Xenorhabdus thuongxuanensis strain 30TX1 (Figure 2; S4 Table).

191

192 Characterization of effect of mitomycin and UV exposure

193 Our results showed that bacterial grow is not disrupted by mitomycin $\mathrm{C}$ induction at early stages 194 of induction (15 and $45 \mathrm{~min}$ ) (Figure 3). Contrastingly, both mitomycin C and UV triggered growth 195 difference in both uninduced cultures and induced cultures in late induction stages (Figure 3). 196 Similarly, after 2 h, bacterial cells density was observed (a slightly lower with cultures exposed to $197 \mathrm{UV})$, but after $10 \mathrm{~h}$ a high mortality rate was observed for the cultures exposed to mitomycin 198 whereas a strong growth reduction was observed for the cultures exposed to UV (Figure 3). 
199 SDS-page electrophoresis showed the presence of phage-particles after $10 \mathrm{~h}$ in lysates of both 200 mitomycin-induced cultures and UV-induced cultures (Figure 4). We also observed four subunits 201 more abundant: 1) below $37 \mathrm{kDa}, 2$ ) above $37 \mathrm{kDa}, 3$ ) above $50 \mathrm{kDa}$ and 4) below $75 \mathrm{kDa}$. We 202 speculate that the subunit above $37 \mathrm{kDa}$ might be the outer membrane porin (OpnP) proteins based 203 on previous reported data (Kim et al., 2003; Leisman et al., 1995; Morales-Soto and Forst, 2011). 204 Three other subunits (between 75-125 Dk) were detected but less abundant. We also observed low 205 signal of phage-particles in lysate of mitomycin-induced cultures after $15 \mathrm{~min}$ and $40 \mathrm{~min}$ of 206 induction.

207

\section{8}

209

210

211

212

213

214

215

216

217

218

219

220

221

222

223

224 head protein, as well as the putative CI repressor (S5 Table). We observed an up-regulation of the gene encoding the major head protein at 2 and $10 \mathrm{~h}$ post mitomycin exposure; and an up-regulation only after $2 \mathrm{~h}$ for the UV exposure (Figure 5). With respect to the CI repressor, an up-regulation 
230 for both mitomycin and UV exposure. However, no significant differential expression of the CI

231 repressor was observed at early time post exposure (15 or 40 minutes).

232 With respect to Xnp4 prophage regions, we quantified the relative expression of the tail 233 fiber protein (S5 Table). We observed an up-regulation of the gene that encodes the tail fiber 234 protein only $2 \mathrm{~h}$ post exposure to either mitomycin or UV. A down-regulation of the gene was 235 observed at $10 \mathrm{~h}$ post-exposure for the UV-induced cultures only.

\section{Discussion}

In this study we expanded existing knowledge on the diversity of integrated phages in of $X$. nematophila genome by describing two additional prophage regions: Xnp3 and Xnp4. The previously described Xnp1 prophage region appears more conserved among Xenorhabdus spp. when compared to the other prophage regions (Figure S1). The three other prophage regions were either not detected in the all the analyzed genomes Xenorhabdus species or presenting more variation. Our study showed that the newly described Xnp3 prophage region is observed in four genomes of Xenorhabdus species and is very similar to complete Mu-like bacteriophage. It is suggested that the acquisition of this prophage region is conserved among some Xenorhabdus species. Our study shows different levels of conservation of these prophage regions among Xenorhabdus species suggesting different selection pressures.

Regarding the mechanisms underlying the induction of phage-like particles in Xenorhabdus, it has been theorized that induced DNA damage could drive the cleavage regulation of the CI repressor present in the P2-remnant prophage locus (Xnp1) leading to the release of phage tail-like particles (Morales-Soto et al., 2012; Thappeta et al., 2020). Although DNA damage mechanisms have not been demonstrated for Xenorhabdus species, the network of genes known as SOS response appears as a near-universal mechanism in bacteria (Fornelos et al., 2016; Lemire et al., 2011; Nanda et al., 2015). This gene regulatory network encompasses genes that are coordinately regulated by the repressor LexA (Fornelos et al., 2016). During DNA damage event, the gene recA is activate and stimulate the self-cleavage of LexA inducing DNA repair responses (Fornelos et exhibit an increase expression of recA, as well as LexA. These mechanisms appear to be activated early after exposure to mitomycin $\mathrm{C}$, (a slight increase was observed within 15 min postinduction. Interestingly, the UV induction condition used in our study (10 min exposure at $\left.6 \mu \mathrm{J} / \mathrm{cm}^{2}\right)$ yielded 
$2615 \mu \mathrm{g} \mathrm{ml}^{-1}$ ) with lower mortality after $10 \mathrm{~h}$ of exposure despite the observed production of phage262 particles. In this induction condition, the lower bacterial cell mortality was associated with the 263 downregulation of the recA regulator (after $10 \mathrm{~h}$ ). In both induction conditions, our study 264 confirmed the involvement of $r e c A$ and $\operatorname{lex} A$ in the DNA damage mechanisms driven by the 265 induction.

266 Regarding the expression of the CI repressor, our results are less clear. For most of the 267 conditions and time points no significant differential expression of the CI repressor for both Xpn1 268 and Xnp3 regions was observed. Although our results suggest low modification of the expression 269 of the CI repressor after induction, further evidence is needed to provide a stronger support for 270 these observations.

271 In this study we did not see evidence for the induction of Xnp2 prophage region by the host 272 SOS response as previously reported (Morales-Soto and Forst, 2011). The Xnp2 genes were hardly 273 detectable by qRT-PCR. It is known that not all integrated phages respond to the LexA system of 274 induction as it has been demonstrated for Phage P2 integrated in E. coli (Bertani and Bertani, 275 1970). It has been demonstrated that the presence of gene coding the cox protein in the prophage 276 region can negatively autoregulate the early operon inhibiting the formation of the lysogenic 277 repressor (Saha et al., 1987). The Xnp2 region contained a gene coding the cox regulator protein 278 which could explained that this prophage region is not induced by mitomycin or UV exposure.

279 Our results showed that the genes contained in Xnp1 prophage region $(G p A$ and $G p W)$ are 280 up regulated after mitomycin and UV exposure. Similarly, Morales-Soto and Forst (2011) 281 previously demonstrated an increase in the expression of genes contained in Xnpl prophage region 282 (Morales-Soto and Forst, 2011). In addition, our study showed that the Xnp1 prophage region is 283 not the only prophage region up regulated during mitomycin and UV exposure The expression of 284 Xnp3 gene (encoding major head protein) and Xnp4 gene (encoding tail fiber protein) exhibited a 285 286 287 288 very similar expression to those quantified for Xnpl genes. Thus, an up-regulation of the three prophage regions was observed in $X$. nematophila during mitomycin and UV exposures.

Previously, it has been shown that deletion of genes in Xnp1 region (coding sheath and fiber protein) in $X$. nematophila affect production of phage tail-like bacteriocin during mitomycin 289 induction (Morales-Soto and Forst, 2011). However, the phage tail-like bacteriocin or xenorhabdicin were not the only phage particles observed as produced by $X$. nematophila cultures exposed to mitomycin, diverse phages particles were previously described in mitomycin $\mathrm{C}$ - 
292 induced cultures of $X$. nematophila (A24/1) (such as bacteriocin with extended sheath, empty 293 sheath, empty phage head, or complete phages) (Boemare et al., 1992). According to our 294 observations, the Xnp3 prophage region sequence, exhibited strong similarities with the complete 295 Mu-like bacteriophage genome sequence. These similarities associated with results of up296 regulation of genes contain in this prophage region after induction suggest that Xnp3 may be 297 involved in phage-like particles, potentially complete phages. Similarly, we might speculate that 298 the Xnp4 may be involved in other phage like-tail particles because only genes encoding tail and 299 fiber protein were identified. However, further experiments including silencing of Xpn3 and Xnp4 300 genes is needed to corroborate this assumption.

301

\section{Conclusions}

303 In this study two integrated prophage regions not previously described in the genome of $X$. 304 nematophila AN6/1 were identified. Although less conserved among Xenorhabdus spp. than the 305 described p1 prophage region, we observed similar prophage region in other Xenorhabdus species. The Xnp3 prophage region very similar to complete Mu-like bacteriophage which was observed in three other available draft Xenorhabdus genomes. In addition, we outline potential genetic mechanisms that occur in $X$. nematophila AN6/1 during mitomycin $\mathrm{C}$ and UV exposure. We showed that mitomycin $\mathrm{C}$ exposure induced an up-regulation of $\operatorname{rec} A$ and $\operatorname{lex} A$ suggesting activation of SOS response as described in other bacteria. Our findings suggest that mitomycin $\mathrm{C}$ and UV exposure lead to up-regulation of three on the four integrated prophages region present in $X$. nematophila genomes. Although our study did not allow for the recognition of an activation role of the newly described prophage region Xnp3 or Xnp4, it suggests that other prophage regions besides Xnpl are inducible. Characterization of the induced particles using plaque formation experiment, as well as, TEM (Transmission Electron Microscopy) studies should be conducted to further characterized the described prophages. Further investigations are also warranted to better understand the diversity and biological role of integrate prophage regions in Xenorhabdus species.

\section{Acknowledgements}

320 We acknowledge Dr. David Baltrus from University of Arizona and Dr. Lise Raleigh from New 321 England Biolabs for their helpful comments on phage biology. 


\section{Author Contributions Statement}

324 EL conceived the experiments and SPS provided supervision and infrastructure support. EL and $325 \mathrm{NC}$ performed the experiments. EL designed the experiments, investigated and analyzed the data. 326 EL and SPS wrote the main manuscript text. All authors reviewed and edited the manuscript. 


\section{Figures caption:}

329

330

331

332

333

334

335

336

337

338

339

340

341

342

343

344

345

346

347

348

349

350

351

352

353

354

355

356

357

358

Figure 1: Prophage regions detected in the Xenorhabdus nematophila strain AN6/1. Detail of the four prophage regions detected and associated with high predicted completeness score according to PHASTER. The annotation of each protein was validated by hmmer and blastp similarities analyses (see detail S3 Table).

Figure 2: Bacteriophages and other Xenorhabdus prophages regions similar to the Xnp3 prophages region. Detail of two $\mathrm{Mu}$-like bacteriophages (SfMu and D108) and prophage regions from other species of Xenorhabdus identified as similar to Xnp3. The annotation of each protein was validated by PHASTER, hmmer and blastp similarities analyses (see detail S4 Table).

Figure 3: Effect of induction by mitomycin and UV exposure on growth of $X$. nematophila AN6/1 cultures. A) Optical density $\left(\mathrm{OD}_{600}\right)$ of $X$. nematophila AN6/1 cultures 15 minutes and 40 minutes after mitomycin induction. B) Optical density $\left(\mathrm{OD}_{600}\right)$ of Xenorhabdus nematophila AN6/1 cultures $2 \mathrm{~h}$ and $10 \mathrm{~h}$ after mitomycin induction and UV induction. A fitted regression line for each condition was calculated by scatterplot (method $=1 \mathrm{~lm}$ ) in R environment.

Figure 4: SDS-PAGE of lysate supernatant from Xenorhabdus nematophila AN6/1 cultures after PEG treatment. Purified lysate of uninduced cultures: lane 1 for culture after 15 min, lane 2 after $40 \mathrm{~min}$ and lane 5 after $10 \mathrm{~h}$. Purified lysate of mitomycin-induced cultures: lane 3, 15 minutes after induction; lane 4, $40 \mathrm{~min}$ after induction; lane 6, $10 \mathrm{~h}$ after induction. Purified lysate of UV-induced cultures: lane 7, $10 \mathrm{~h}$ after induction. Lane L are Precision Plus Protein standard dual color (Bio-Rad). The putative outer membrane porin (OpnP) proteins is indicated by an arrow.

Figure 5: Relative expression of $\operatorname{lex} A, \operatorname{rec} A$ and prophages genes of mitomycin-induced and UV-induced Xenorhabdus nematophila AN6/1 cultures. Relative expression of each gene were normalized using gyrA as housekeeping gene and uninduced Xenorhabdus nematophila AN6/1 cultures were used as calibrator. The log base 2 of relative expression were plot and statistical tests were performed on relative expression value (see detail S5 Table). Abbreviation: GpA: replication gene $\mathrm{A} ; \mathrm{GpW}$ : baseplate assembly protein $\mathrm{W}$; CI: putative CI repressor gene. 


\section{References:}

360 Arndt, D., Grant, J.R., Marcu, A., Sajed, T., Pon, A., Liang, Y., Wishart, D.S., 2016. PHASTER:

361

362

363

364

365

366

367

368

369

370

371

372

373

374

375

376

377

378

379

380

381

382

383

384

385

386

387

388

389

a better, faster version of the PHAST phage search tool. Nucleic Acids Res. 44, W16-W21. https://doi.org/10.1093/nar/gkw387

Baluch, J., Sussman, R., 1978. Correlation between UV dose requirement for lambda bacteriophage induction and lambda repressor concentration. J. Virol. 26, 595-602. https://doi.org/10.1128/jvi.26.3.595-602.1978

Bertani, L.E., Bertani, G., 1970. Preparation and characterization of temperate, non-inducible bacteriophage P2 (host: Escherichia coli). J. Gen. Virol. 6, 201-212. https://doi.org/10.1099/0022-1317-6-2-201

Bobay, L.-M., Touchon, M., Rocha, E.P.C., 2014. Pervasive domestication of defective prophages by bacteria. PNAS 111, 12127-12132. https://doi.org/10.1073/pnas.1405336111

Boemare, N., 2002. Interactions between the partners of the entomopathogenic bacterium nematode complexes, Steinernema-Xenorhabdus and Heterorhabditis-Photorhabdus. Nematology 4, 601-603. https://doi.org/10.1163/15685410260438863

Boemare, N.E., Boyer-Giglio, M.H., Thaler, J.O., Akhurst, R.J., Brehelin, M., 1992. Lysogeny and bacteriocinogeny in Xenorhabdus nematophilus and other Xenorhabdus spp. Appl. Environ. Microbiol. 58.

Camacho, C., Coulouris, G., Avagyan, V., Ma, N., Papadopoulos, J., Bealer, K., Madden, T.L., 2009. BLAST+: Architecture and applications. BMC Bioinformatics 10. https://doi.org/10.1186/1471-2105-10-421

Ciezki, K., Murfin, K., Goodrich-Blair, H., Stock, S.P., Forst, S., 2017. R-type bacteriocins in related strains of Xenorhabdus bovienii: Xenorhabdicin tail fiber modularity and contribution to competitiveness. FEMS Microbiol. Lett. https://doi.org/10.1093/femsle/fnw235

Dunn, O.J., 1964. Multiple Comparisons Using Rank Sums. Technometrics 6, 241-252. https://doi.org/10.1080/00401706.1964.10490181

Flores-Lara, Y., Renneckar, D., Forst, S., Goodrich-Blair, H., Stock, P., 2007. Influence of nematode age and culture conditions on morphological and physiological parameters in the bacterial vesicle of Steinernema carpocapsae (Nematoda: Steinernematidae). J. Invertebr. Pathol. 95, 110-118. https://doi.org/10.1016/j.jip.2007.01.006 
390 Fornelos, N., Browning, D.F., Butala, M., 2016. The Use and Abuse of LexA by Mobile Genetic 391 Elements. Trends Microbiol. https://doi.org/10.1016/j.tim.2016.02.009

392 Fox, J., Weisberg, S., 2019. An R Companion to Applied Regression, third edit. ed. SAGE 393 Publications Inc.

394 Griffin, A.S., West, S.A., Buckling, A., 2004. Cooperation and competition in pathogenic 395 bacteria. Nature 430, 1024-1027. https://doi.org/10.1038/nature02744

396 Harrison, E., Brockhurst, M.A., 2017. Ecological and Evolutionary Benefits of Temperate

397 398 399 400 401 402 403 404 405 406 407 408 409 410

411

412

413

414

415

416

417

418

419

420 Phage: What Does or Doesn't Kill You Makes You Stronger. BioEssays. https://doi.org/10.1002/bies.201700112

Kim, D.J., Boylan, B., George, N., Forst, S., 2003. Inactivation of ompR promotes precocious swarming and flhDC expression in Xenorhabdus nematophila. J. Bacteriol. 185, 52905294. https://doi.org/10.1128/JB.185.17.5290-5294.2003

Kim, S.K., Flores-Lara, Y., Patricia Stock, S., 2012. Morphology and ultrastructure of the bacterial receptacle in Steinernema nematodes (Nematoda: Steinernematidae). J. Invertebr. Pathol. 110, 366-374. https://doi.org/10.1016/j.jip.2012.04.011

Leisman, G.B., Waukau, J., Forst, S.A., 1995. Characterization and environmental regulation of outer membrane proteins in Xenorhabdus nematophilus. Appl. Environ. Microbiol. 61, 200204. https://doi.org/10.1128/aem.61.1.200-204.1995

Lemire, S., Figueroa-Bossi, N., Bossi, L., 2011. Bacteriophage crosstalk: Coordination of prophage induction by Trans-Acting antirepressors. PLoS Genet. 7. https://doi.org/10.1371/journal.pgen.1002149

Morales-Soto, N., Forst, S.A., 2011. The xnp1 p2-like tail synthesis gene cluster encodes xenorhabdicin and is required for interspecies competition. J. Bacteriol. 193, 3624-3632. https://doi.org/10.1128/JB.00092-11

Morales-Soto, N., Gaudriault, S., Ogier, J.C., Thappeta, K.R.V., Forst, S., 2012. Comparative analysis of P2-type remnant prophage loci in Xenorhabdus bovienii and Xenorhabdus nematophila required for xenorhabdicin production. FEMS Microbiol. Lett. 333, 69-76. https://doi.org/10.1111/j.1574-6968.2012.02600.x

Nanda, A.M., Thormann, K., Frunzke, J., 2015. Impact of spontaneous prophage induction on the fitness of bacterial populations and host-microbe interactions. J. Bacteriol. https://doi.org/10.1128/JB.02230-14 
421 Pfaffl, M.W., 2001. A new mathematical model for relative quantification in real-time RT-PCR.

422 Nucleic Acids Res. 29, E45. https://doi.org/10.1093/nar/29.9.e45

423 Poinar, G.O., Hess, R., Thomas, G., 1980. Isolation of defective bacteriophages from

424 Xenorhabdus spp. (Enterobacteriaceae). IRCS Med. Sci. Cell Membr. Biol. 141.

425 Potter, S.C., Luciani, A., Eddy, S.R., Park, Y., Lopez, R., Finn, R.D., 2018. HMMER web

426 server: 2018 update. Nucleic Acids Res. 46, W200-W204.

427 https://doi.org/10.1093/nar/gky448

428 Saha, S., Lundqvist, B., Haggård-Ljungquist, E., 1987. Autoregulation of bacteriophage P2

429 repressor. EMBO J. 6, 809-814. https://doi.org/10.1002/j.1460-2075.1987.tb04823.x

430 Selva, L., Viana, D., Regev-Yochay, G., Trzcinski, K., Corpa, J.M., Lasa, Í., Novick, R.P.,

431 Penadés, J.R., 2009. Killing niche competitors by remote-control bacteriophage induction.

432 Proc. Natl. Acad. Sci. U. S. A. 106, 1234-1238. https://doi.org/10.1073/pnas.0809600106

433 Stock, S.P., 2019. Partners in crime: symbiont-assisted resource acquisition in Steinernema

434 entomopathogenic nematodes. Curr. Opin. Insect Sci.

435 https://doi.org/10.1016/j.cois.2018.10.006

436 Stock, S.P., Blair, H.G., 2008. Entomopathogenic nematodes and their bacterial symbionts: The 437 inside out of a mutualistic association. Symbiosis 46, 65-75.

438 Takebe, H., Ichikawa, H., Iwo, K., Kondo, S., 1967. Phage induction by ultraviolet radiation in 439 strains of Escherichia coli possessing and lacking dark repair capacity. Virology 33, 638440 649. https://doi.org/10.1016/0042-6822(67)90064-5

441 Thaler, J.O., Baghdiguian, S., Boemare, N., 1995. Purification and characterization of 442 xenorhabdicin, a phage tail-like bacteriocin, from the lysogenic strain F1 of Xenorhabdus 443 nematophilus. Appl. Environ. Microbiol. 61.

444 Thappeta, K.R.V., Ciezki, K., Morales-Soto, N., Wesener, S., Goodrich-Blair, H., Stock, S.P., 445 Forst, S., 2020. R-type bacteriocins of xenorhabdus bovienii determine the outcome of 446 interspecies competition in a natural host environment. Microbiol. (United Kingdom) 166, 447 1074-1087. https://doi.org/10.1099/mic.0.000981

448 Vacheron, J., Heiman, C.M., Keel, C., 2021. Live cell dynamics of production, explosive release 449 and killing activity of phage tail-like weapons for Pseudomonas kin exclusion. Commun.

$450 \quad$ Biol. 4. https://doi.org/10.1038/s42003-020-01581-1

451 
453 Supplementary files caption:

454

455 Figure S1. Phylogenetic analyses of prophage genes.

456 A) Phylogeny of Phage-related minor tail protein present in Xnp1, Xnp2 and Xnp3. Analysis of

45776 sequences with 1,919 amino-acid sites. B) Phylogeny of 3 proteins (tail protein and fiber

458 protein) present in Xnp4 prophage region and orthologous proteins present in other bacteria.

459 Analysis of 8 sequences with 1,286 amino-acid sites.

460 The phylogenetic analyses were inferred using ML inference using iq-tree. Nodes are associated 461 with bootstrap values based on 1000 replicates. The best-fit model calculated using ModelFinder 462 according to the AIC index : JTT $+\mathrm{F}+\mathrm{I}+\mathrm{G} 4$ for $\mathrm{A}$ and $\mathrm{LG}+\mathrm{F}+\mathrm{G} 4$ for $\mathrm{B}$. The accession number of 463 the studied proteins are indicates in bracket after the species names. The scale bar indicates the 464 distance in substitutions per nucleotide.

465

466

467

Table S1. Information about the design and optimization of primers for $q R T-P C R$ validation.

468

469

470

Table S2. Summary of potential prophages regions detected in the genome of Xenorhabdus nematophila strain AN6/1 (NZ_LN681227) using PHASTER.

471

472

473

474

Table S3. List of annotation of genes present in the Xnp1, Xnp2, Xnp3 and Xnp4 prophage regions identified in the genome of Xenorhabdus nematophila strain AN6/1

475 (NZ_LN681227).

478

479

480

481

482

Table S4. Detail of annotation of genes present in the bacteriophages and prophages region of Xenorhabdus spp. identified as closely related to the Xnp3 prophage region of $X$. nematophila AN6/1.

Table S5. Summary of statistically analyses performed on the relative expression established by qRT-PCR for the targeted genes associated with different induction conditions at different time points.

483 All the tests were performed in the $\mathrm{R}$ environment. For all the data, we tested if the data were 
484 normally distributed (Shapiro-Wilk normality test), present common variance (Levene's test) and 485 were independent group (Chi-squared test). These three assumptions were not validated, thus 486 non-parametric Kruskal-wallis rank sum associated with multiple pairwise comparisons using the 487 dunn's test was used. 


\section{Figure 1}

\section{Figure 1}

\section{Prophages regions detected in the Xenorhabdus nematophila strain AN6/1}
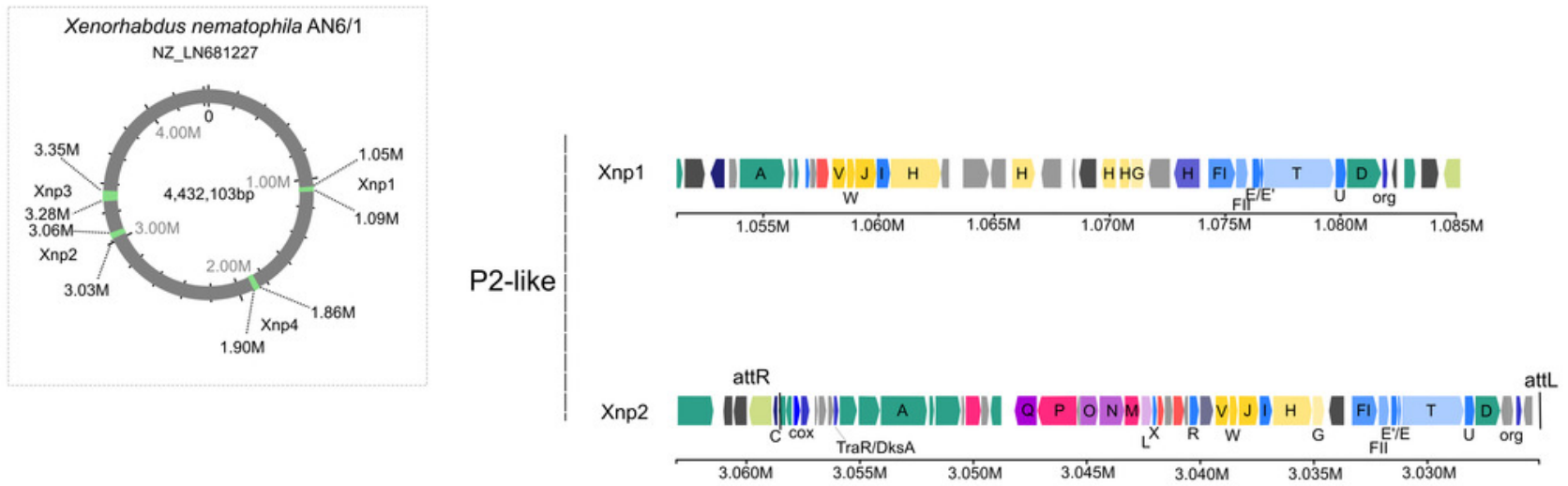

Mu-like
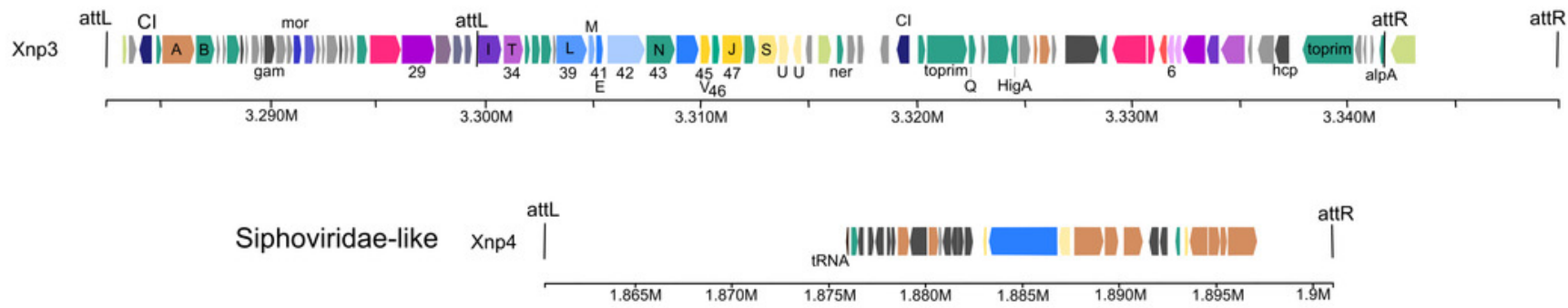

Annotation legend:

\begin{tabular}{lll}
\hline Phage-like protein & Capsid protein \\
Protease & Head completion protein \\
Terminase & Head-tail connector \\
Portal protein & Virion morphogenesis protein \\
& Head morphogenesis protein
\end{tabular}

Tail protein
Tail sheath protein
Tail tube protein
Tail tape measure protein

Baseplate protein

Tail collar-fiber protein

Tail Fiber assembly protein

Repressor

Regulator

Lysis

Transposase

Integrase

Hypothetical protein

Other 


\section{Figure 2}

\section{Figure 2}

\section{Bacteriophages and other Xenorhabdus prophages regions similar to the Xnp3 prophages}

\section{region}

Annotation legend:

$\begin{array}{lll}\text { Protease } & \text { Capsid protein } \\ \text { Terminase } & \text { Head completion protein } \\ \text { Portal protein } & \text { Head-tail connector } \\ \text { Phage-like protein } & \text { Head morphogenesis protein } \\ & \text { Virion morphogenesis protein }\end{array}$

Tail protein Tail sheath protein

Tail tube protein

Tail tape measure protein
Baseplate protein Tail collar-fiber protein Tail Fiber assembly protein

Repressor

Regulator
Lysis

Transposase

Integrase

Hypothetical protein

Other

Bacteriophage SfMu Cl NC_027382

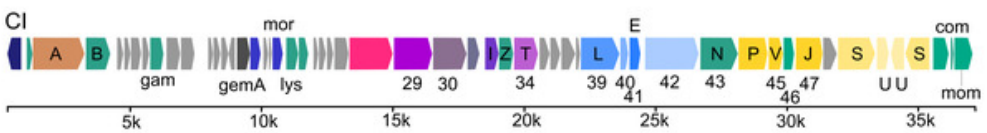

Bacteriophage D108 NC_013594

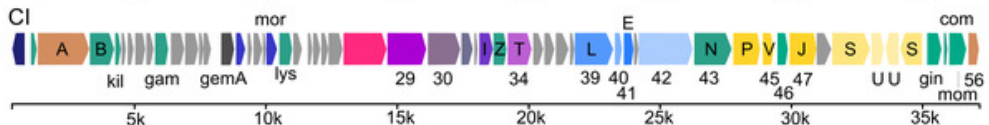

Xenorhabdus nematophila AN6/1

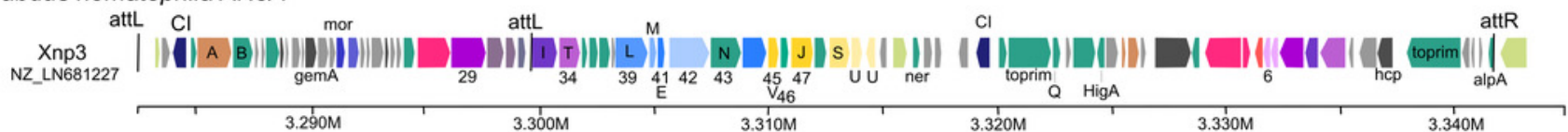

Xenorhabdus cabanillasii JM26

contig Xcab 8 NZ_NJGH01000008

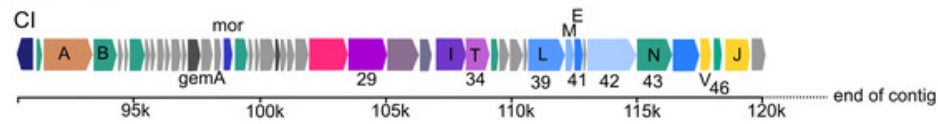

Xenorhabdus miraniensis DSM17902

contig Xmir_5 NZ_NITZ01000005

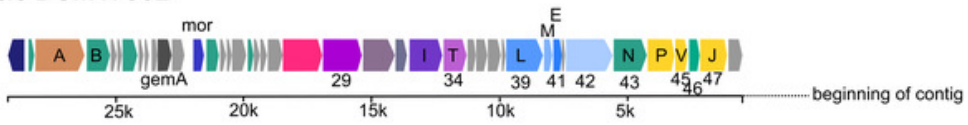

Xenorhabdus thuongxuanensis 30TX1

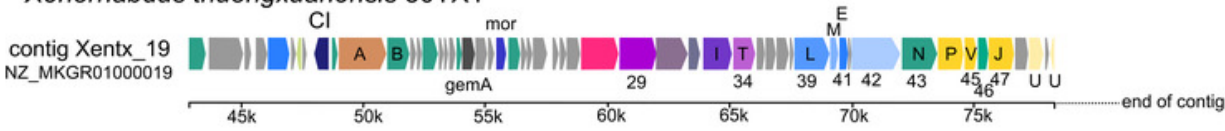


Figure 3

Effect of induction by mitomycin and UV exposure on growth of $X$. nematophila AN6/1 cultures

A) Optical density $\left(\mathrm{OD}_{600}\right)$ of $X$. nematophila AN6/1 cultures 15 minutes and 40 minutes after mitomycin induction. B) Optical density $\left(\mathrm{OD}_{600}\right)$ of Xenorhabdus nematophila AN6/1 cultures 2 $\mathrm{h}$ and $10 \mathrm{~h}$ after mitomycin induction and UV induction. A fitted regression line for each condition was calculated by scatterplot (method $=\mathrm{Im}$ ) in R environment.
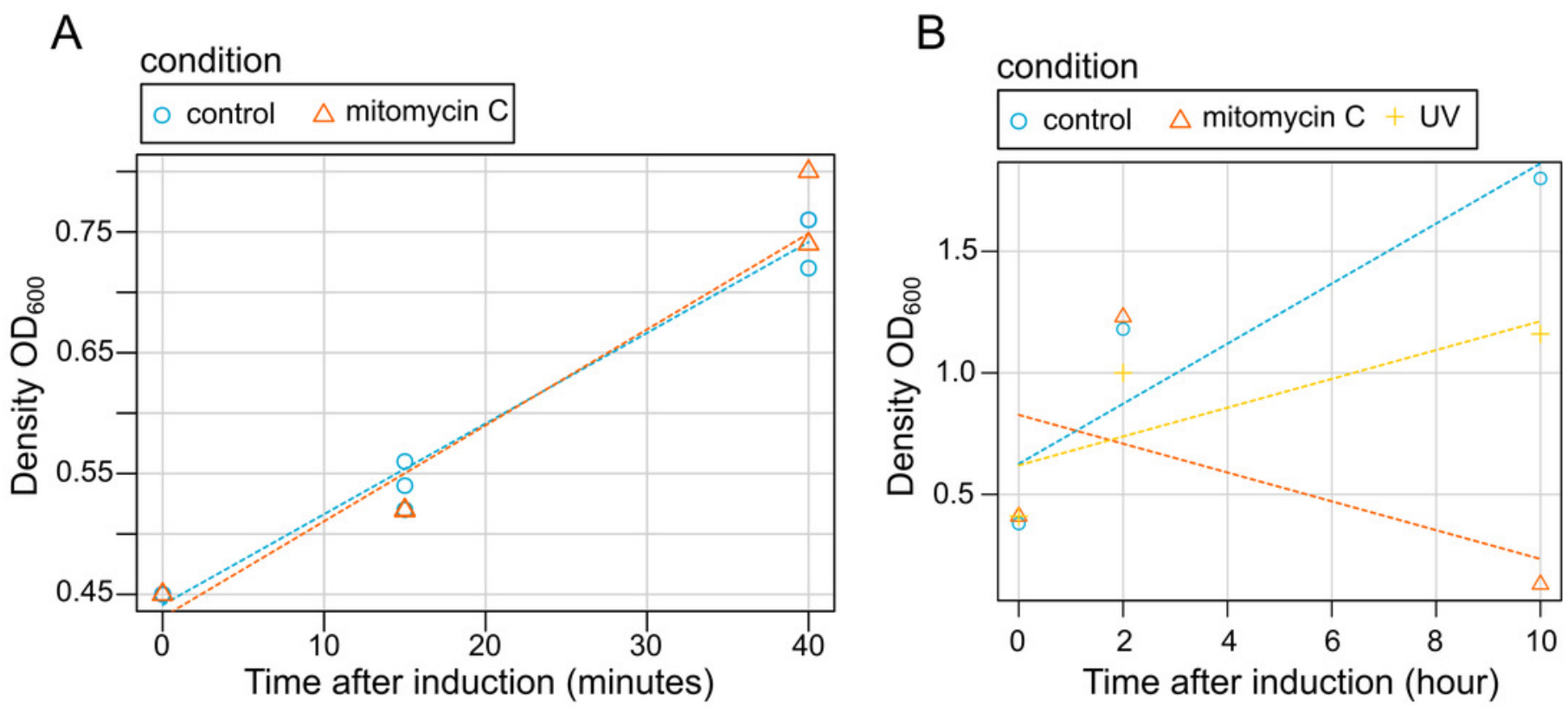
Figure 4

Figure 4

SDS-PAGE of lysate supernatant from Xenorhabdus nematophila AN6/1 cultures after PEG treatment 


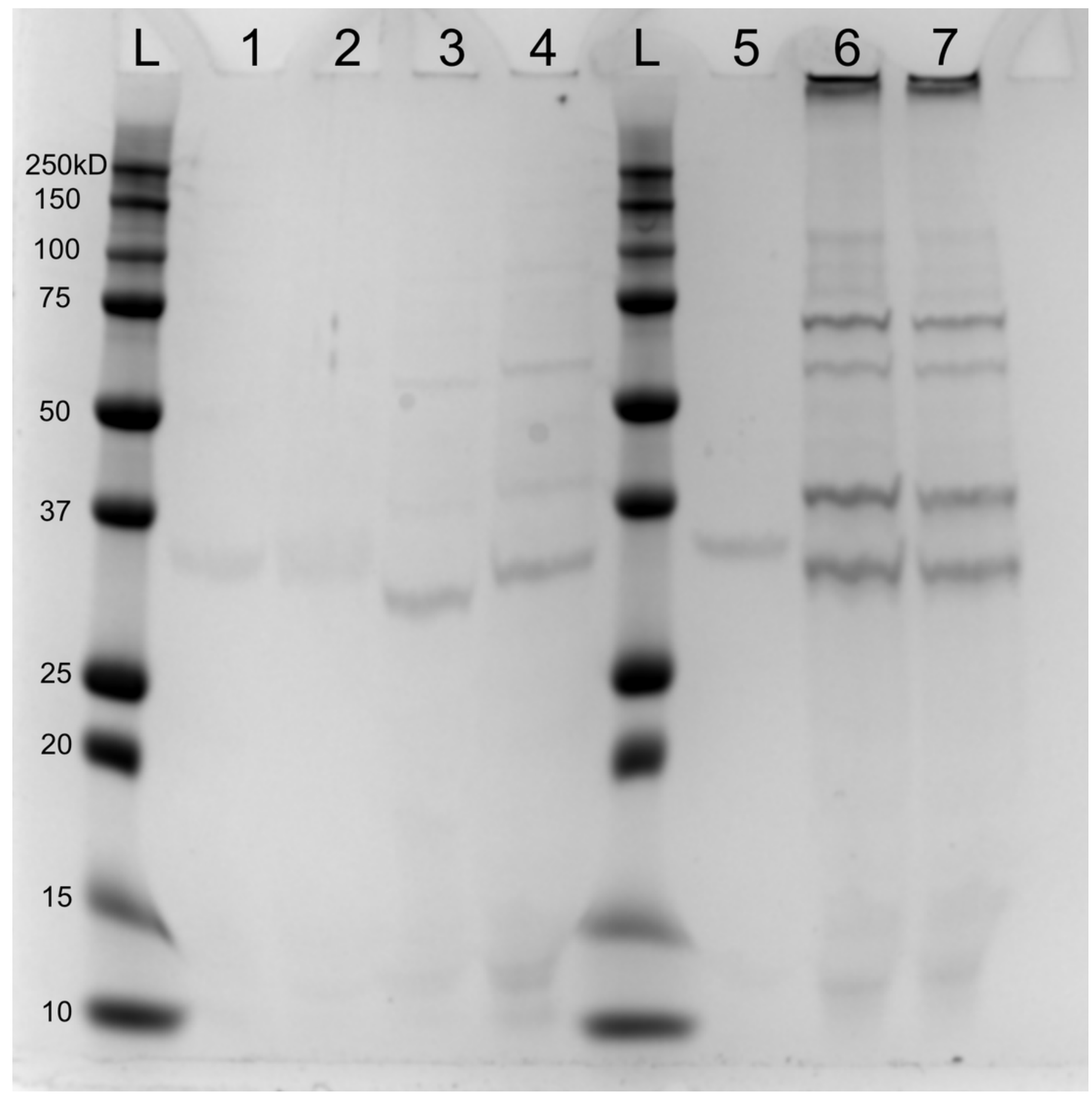




\section{Figure 5}

Relative expression of lexA, recA and prophages genes of mitomycin-induced and UVinduced Xenorhabdus nematophila AN6/1 cultures.

Relative expression of each gene were normalized using gyrA as housekeeping gene and uninduced Xenorhabdus nematophila AN6/1 cultures were used as calibrator. The log base 2 of relative expression were plot and statistical tests were performed on relative expression value (see detail S5 Table). Abbreviation: GpA: replication gene A; GpW : baseplate assembly protein W; Cl: putative $\mathrm{Cl}$ repressor gene. 

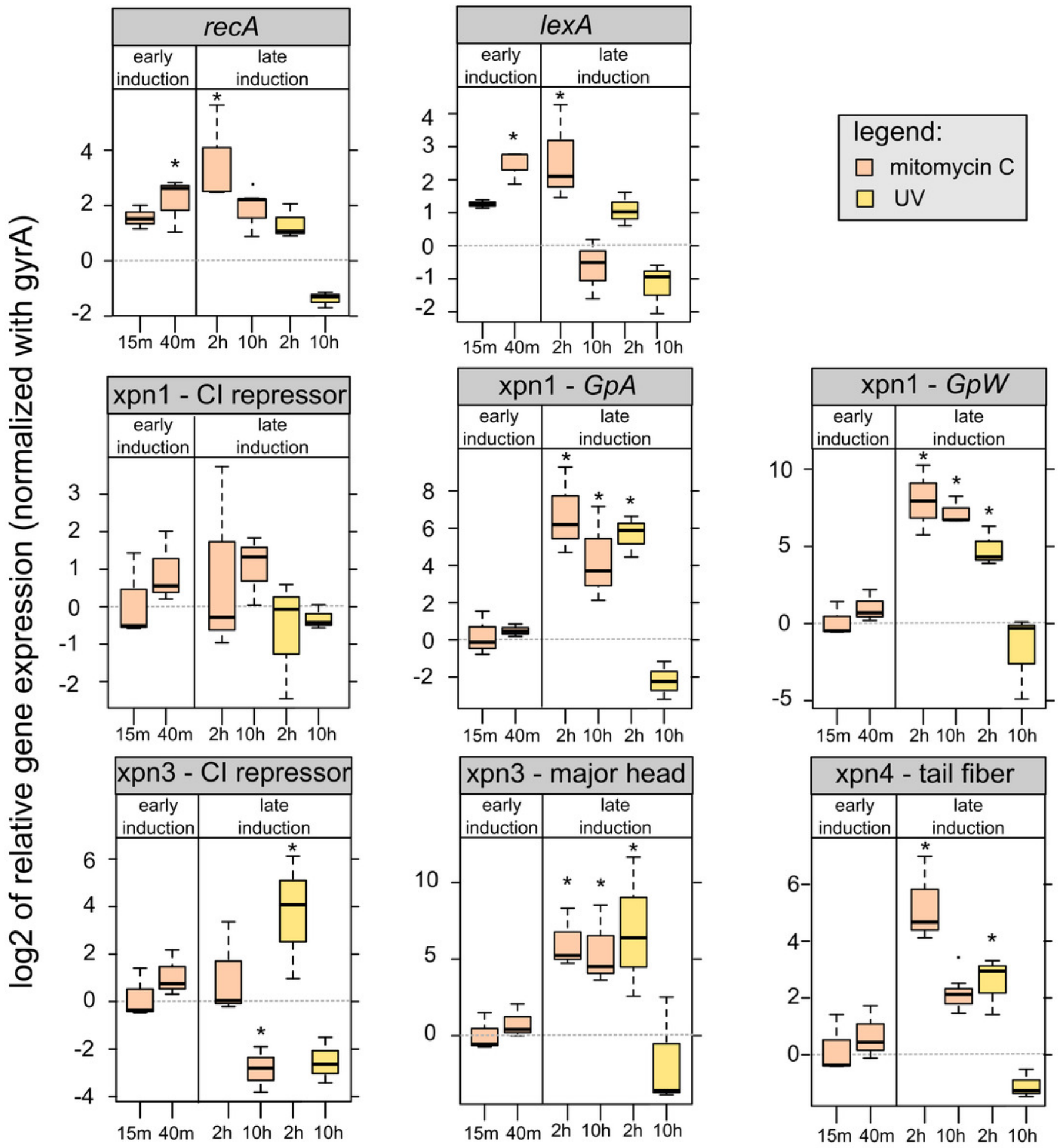

Time after induction 